\title{
PM Assisted, Brushless Wound Rotor Synchronous Machine
}

\author{
Qasim Ali ${ }^{1}$, Shahid Atiq ${ }^{2}$, Thomas A. Lipo ${ }^{3}$, and Byung-il Kwon ${ }^{1 *}$ \\ ${ }^{1}$ Hanyang University, Ansan 15588, Korea \\ ${ }^{2}$ The Islamia University of Bahawalpur, Bahawalpur 63100, Pakistan \\ ${ }^{3}$ Florida State University, Tallahassee FL 32306, USA
}

(Received 18 May 2016, Received in final form 29 July 2016, Accepted 5 August 2016)

\begin{abstract}
This paper presents a new permanent magnet (PM) assisted topology for a recently introduced brushless wound rotor synchronous machine (BL-WRSM) [1]. The BL-WRSM had a dual-inverter configuration for generating a composite magneto motive force (MMF) with a fundamental component and a subharmonic component. The subharmonic component of the MMF is used for brushless excitation of the rotor. In this paper, additional PMs were introduced on the rotor of the BL-WRSM, making it a hybrid BL-WRSM. We also discussed the flux weakening operation for the hybrid BL-WRSM. The hybrid BL-WRSM offered advantages for starting the machine and provided better performance under full-load conditions. The finite element method (FEM) was used to analyze the performance of the hybrid BL-WRSM, and we compared its performance with BL-WRSM. Finally, prototypes were built with and without the PM-assistance, and experiments were conducted to demonstrate their performance.
\end{abstract}

Keywords : brushless excitation, synchronous machine, hybrid excitation, flux weakening

\section{Introduction}

Permanent magnet (PM) machines have been the most commonly used machines in recent years. Their popularity is due to their high efficiency under normal loads and high torque-to-volume ratio. However, researchers are developing alternatives due to the high cost and limited availability of PM machines; these alternatives include PM-free machines or machines with a lower amount of PM [2]. Hybrid excited machines with both PMs and electrical excitations are discussed in [3]. A hybrid double-excited synchronous machine has been introduced for direct drive applications [4].

Wound-rotor synchronous machines (WRSMs) are magnet-free synchronous machines that utilize rotor windings to generate flux. The effectiveness of WRSMs was analyzed in [5]. In [6], a WRSM was designed and analyzed for a belt-driven e-Assist system, and its performance was compared with that of an interior permanent magnet synchronous motor (IPMSM). It was shown that a WRSM is 5\% more efficient at high speeds

(C)The Korean Magnetics Society. All rights reserved.

*Corresponding author: Tel: +82-31-400-5165

Fax: +82-31-409-1277, e-mail: bikwon@hanyang.ac.kr than an IPMSM. The core loss and copper loss of the WRSM was studied in [7]. The WRSM requires brushes and slip rings, which need maintenance and can experience losses and sparking. Different brushless techniques were discussed in [8-11] with a goal of overcoming the problems related to brushes and slip rings. In [8], additional windings were placed on the stator to achieve brushless operation, but this increased the overall area of the stator. Higher harmonics of the stator MMF were used for rotor excitation in [9] and [10], and this method resulted in brushless operation at the cost of increased losses due to the higher harmonic generation. In [11], brushless operation was achieved by generating a third harmonic zero sequence current in the stator windings by operating switches that were connected in parallel with each phase winding.

Recently, a BL-WRSM topology based on a dualinverter scheme was introduced in [1], shown in Fig. 1. The idea was to generate an additional subharmonic (along with the fundamental component) in the stator magneto motive force (MMF). This was achieved by injecting currents of different magnitudes in two portions of the stator windings via dual inverter operation.

The winding distribution of the BL-WRSM is shown in Fig. 2 along with the stator MMF. The plot shows the case of maximum current in phase A and currents in A2, 


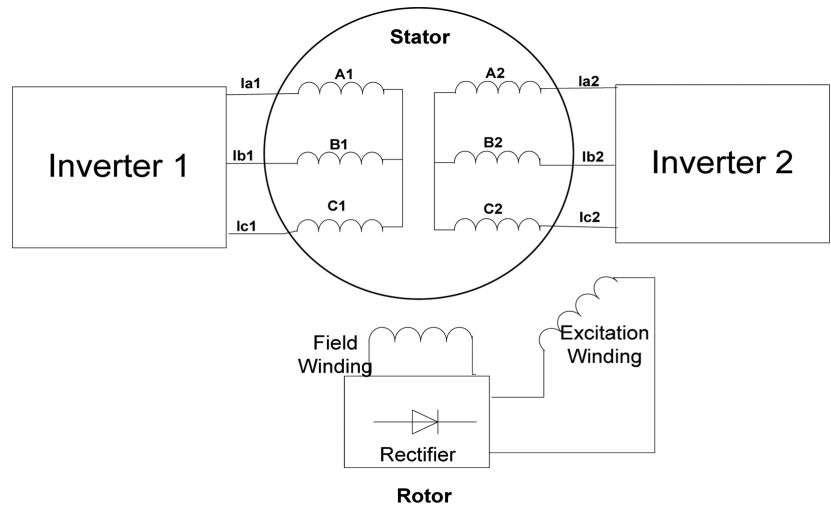

Fig. 1. Brushless WRSM topology.

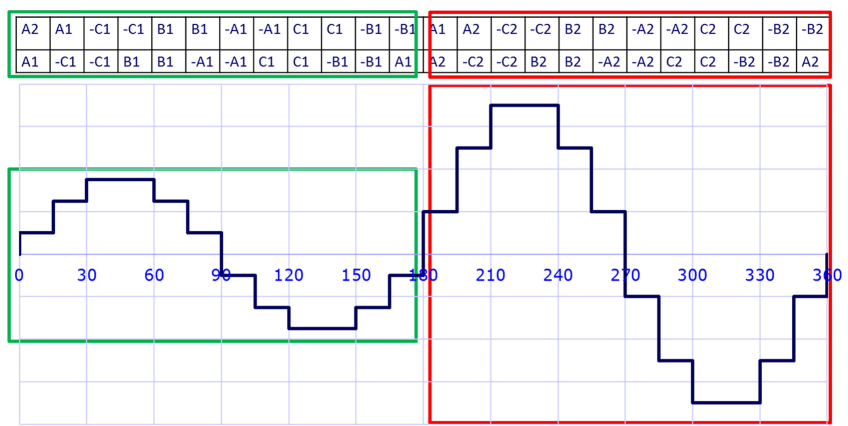

Fig. 2. (Color online) MMF plot for 24 slot stator windings.

$\mathrm{B} 2$, and $\mathrm{C} 2$ are twice the magnitude of the currents in $\mathrm{A} 1$, $\mathrm{B} 1$, and $\mathrm{C} 1$. The composite waveform of the MMF reflects the presence of two dominant components: a fundamental component and a subharmonic at half the fundamental frequency. These two components are responsible for the generation of a 4-pole field and a 2-pole field, respectively. The rotor consists of two separate windings, field and excitation windings, and a rotating rectifier, as shown in Fig. 1. The subharmonic component of the stator MMF was induced on the excitation winding, which further supplied DC current to the field windings through the rectifier. In this way, the field winding was linked to the fundamental component of the stator MMF.

This BL-WRSM topology offered brushless operation through the use of a subharmonic MMF component, which provided advantages over higher-harmonic excitation methods. However, it required additional aid to start the machine because it provides no initial source of rotor flux. Also, the torque density is low compared to those of PM machines. The BL-WRSM topology has no electrical source directly connected to the rotor field windings, so there will be no current flowing in the windings for starting the machine. There are several possible solutions. Generally, damper windings are used to start synchronous machines, but in this case we are already using the two windings in the rotor, therefore it is not a suitable solution to add another winding to start the machine.

The machine can be started on the reluctance torque if it is designed to have a high saliency ratio, but a high saliency ratio will also result in a high torque ripple, which is undesirable for a lot of applications.

In this paper, a hybrid BL-WRSM topology is introduced with a similar stator configuration to that of [1], but the rotor has PMs on it to assist with starting the machine. The rotor with both PMs and windings is a series hybrid rotor, where the flux from the windings passes through the PMs. The hybrid BL-WRSM imparts the advantages of improved starting ability and increased torque density in the machine. The hybrid BL-WRSM is discussed in detail in the next section. We then discuss the flux weakening operation in the machine. Finally, an experimental analysis of the BL-WRSM and the hybrid BL-WRSM are presented.

\section{Proposed Hybrid BL-WRSM Topology}

The proposed hybrid topology with windings and PMs on the rotor can help ensure smooth startup of the

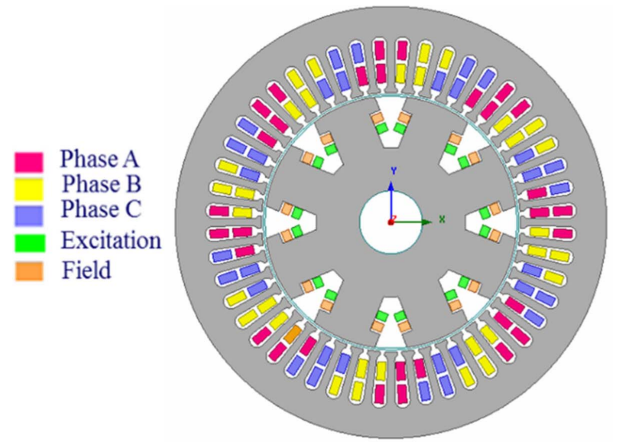

(a)

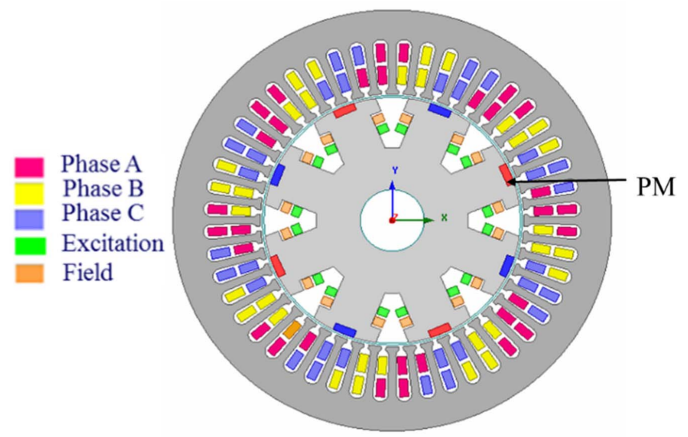

(b)

Fig. 3. (Color online) 2D models for 8-pole machine: (a) BL-WRSM and (b) hybrid BL-WRSM. 


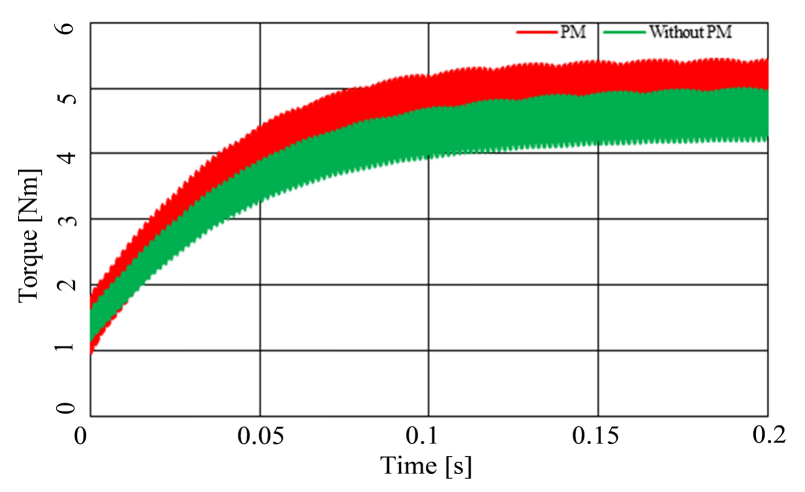

Fig. 4. (Color online) Electromagnetic torque for the BLWRSM and hybrid BL-WRSM.

machine without any external aid. The machine starts as a permanent magnet synchronous machine (PMSM) because there is no induced current in the rotor excitation winding at startup. Gradually, currents are induced in the rotor excitation winding and are rectified and supplied to the rotor field winding. At this stage, the machine operates like a hybrid machine, with flux from both the PMs and windings. Two-dimensional models of the 8-pole BLWRSM and hybrid BL-WRSM are shown in Fig. 3. For the hybrid BL-WRSM case, the rotor and stator windings are similar to the winding configurations shown in Fig. 1. The new feature is the additional PMs in the rotor teeth.

For the initial analysis, 2D finite element analysis (FEA) was performed in ANSYS Maxwell to simulate both BLWRSM and hybrid BL-WRSM. The electromagnetic torque values for the BL-WRSM and hybrid BL-WRSM are shown in Fig. 4. The average torque of the hybrid BLWRSM $(5.01 \mathrm{Nm})$ was higher than that of the BLWRSM model $(4.74 \mathrm{Nm})$. The torque ripple of the hybrid BL-WRSM $(16.03 \%)$ was lower than that of the BLWRSM model (16.29\%).

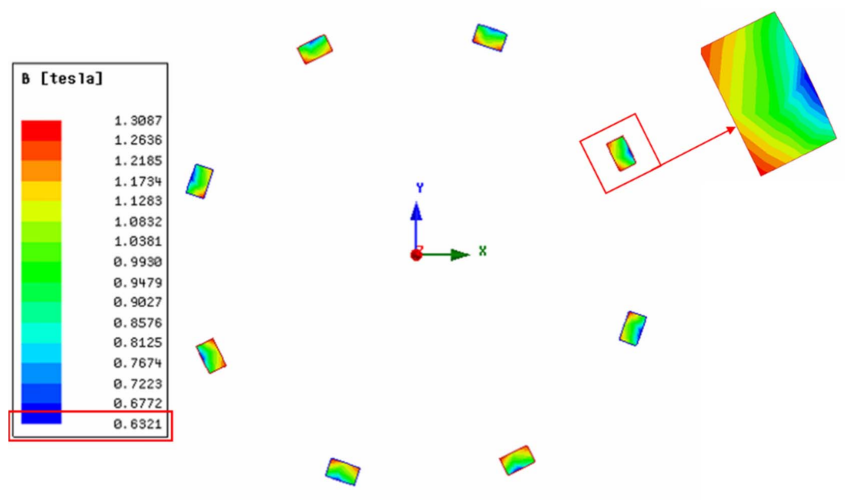

Fig. 5. (Color online) Magnetic field density B for the rotor magnets.
The magnet used in the hybrid BL-WRSM is neodymium iron boron $(\mathrm{NdFeB})$ because of its high performance. The width of the PM was $6 \mathrm{~mm}$, while the height was $3.6 \mathrm{~mm}$. The strength of the magnets in the operating range was estimated by their magnetic flux density (B). The flux density B is plotted for the PMs under the rated currents, as shown in Fig. 5. The maximum value of B was 1.3087 $\mathrm{T}$ and the minimum value was $0.6321 \mathrm{~T}$, which is well within the safe operating range of $\mathrm{NdFeB}$ magnets.

\section{Flux Weakening Operation}

In the BL-WRSM topology of [1], there should be a difference between the current magnitudes in two portions of the stator windings in order to generate a subharmonic to excite the rotor.

$$
\begin{aligned}
& \left\{\begin{array}{l}
i_{a 1}=I_{1} \sin \omega_{e} t \\
i_{b 1}=I_{1} \sin \left(\omega_{e} t-\frac{2 \pi}{3}\right) \\
i_{c 1}=I_{1} \sin \left(\omega_{e} t+\frac{2 \pi}{3}\right)
\end{array}\right\} \\
& \left\{\begin{array}{l}
i_{a 2}=m \times I_{1} \sin \omega_{e} t \\
i_{b 2}=m \times I_{1} \sin \left(\omega_{e} t-\frac{2 \pi}{3}\right) \\
i_{c 2}=m \times I_{1} \sin \left(\omega_{e} t+\frac{2 \pi}{3}\right)
\end{array}\right\} \\
& m=i_{a 2} / i_{a 1}
\end{aligned}
$$

Equations (1) and (2) show the currents in two portions of the stator windings. Equation (3) defines the factor $m$, which is the ratio of the magnitudes of the two inverter currents. The current induced in the rotor excitation wind-

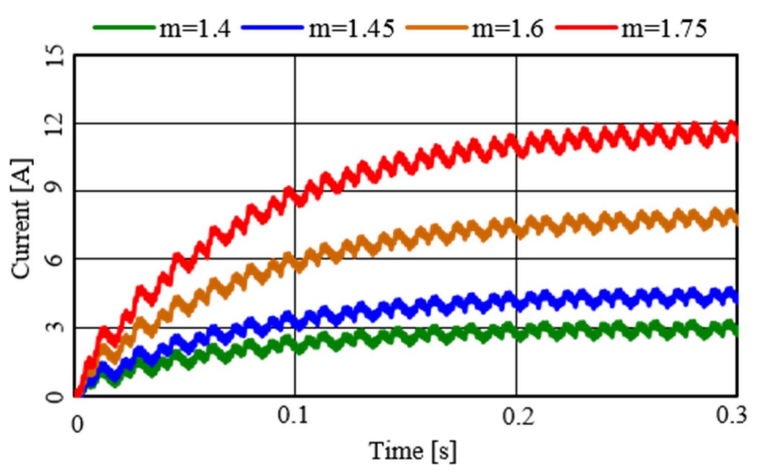

Fig. 6. (Color online) Rectified current supplied to rotor field winding for different values of $m$. 
ing due to the subharmonic component is rectified and fed to the rotor field winding. Figure 6 shows the rectified current fed to the rotor field windings for different values of $m$.

The induced currents in the rotor windings decreased as the difference between the currents in the two portions of the stator winding decreased (smaller $m$ ). This suggests that the voltages induced on the stator due to rotor flux will also decrease as field currents decrease. This indicates that the speed of the machine can be increased beyond its rated speed (with reduced torque) by keeping the terminal voltages constant at the stator side, which is the same as flux weakening in the PM machine.

For the hybrid BL-WRSM, we can employ this method until $m=1$, when the currents in the two stator portions are equal. At this point, no voltage will be induced on the rotor excitation winding, and the machine will only operate due to the PMs on the rotor.

\section{Experimental Verification}

Prototype machines for the proposed hybrid BL-WRSM and BL-WRSM were manufactured, and experimental verification was performed. As the stators of the two models are the same, only a single stator was built. Two separate rotors were built for the two models. The specifications and dimensions of the machines are provided in Table 1.

Figure 7(a) shows the stator core laminations and completed stator with double layer windings and 48 slots. Figure $7(\mathrm{~b})$ shows the two rotor core stack laminations. Figure 7(c) shows both the rotors having excitation windings, field windings, rotating rectifiers and additional PMs for the PM assisted rotor. Figure 7(d) shows the complete experimental setup with a prototype machine connected

Table 1. Experimental Machine Specifications.

\begin{tabular}{lcc}
\hline \hline \multicolumn{1}{c}{ Item } & Units & Value \\
\hline Operating speed & $\mathrm{rpm}$ & 900 \\
Stator outer diameter & $\mathrm{mm}$ & 230 \\
Stator inner diameter & $\mathrm{mm}$ & 95 \\
Air gap length & $\mathrm{mm}$ & 1 \\
Machine axial length & $\mathrm{mm}$ & 80 \\
Number of poles & - & 8 \\
Number of stator slots & - & 48 \\
Magnet Height & $\mathrm{mm}$ & 4 \\
Magnet width & $\mathrm{mm}$ & 6 \\
Conductors per stator slot & - & 40 \\
Conductor diameter (stator and rotor & $\mathrm{mm}$ & 1.1 \\
winding) & & \\
\hline
\end{tabular}

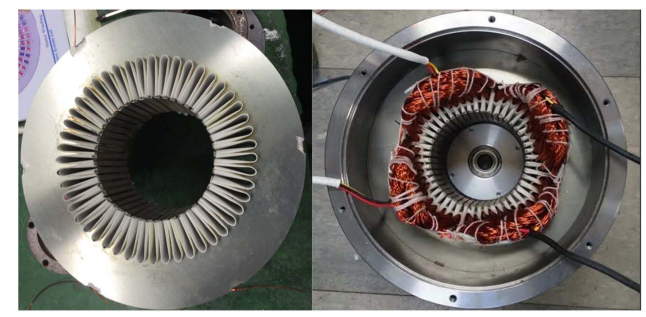

(a)

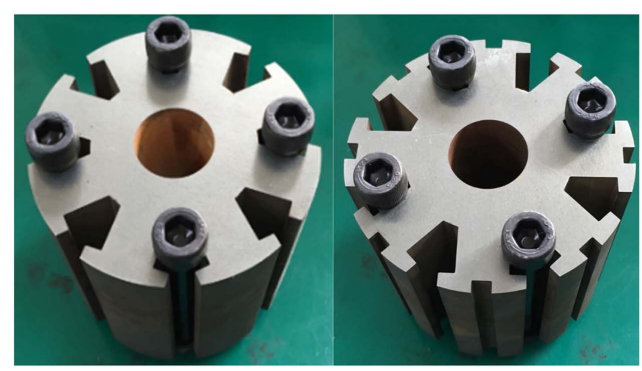

(b)

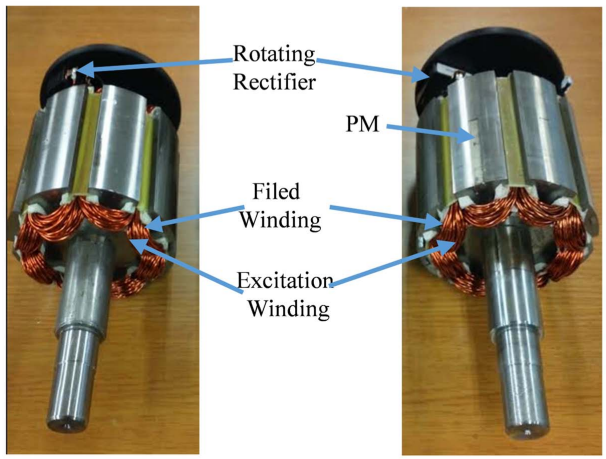

(c)

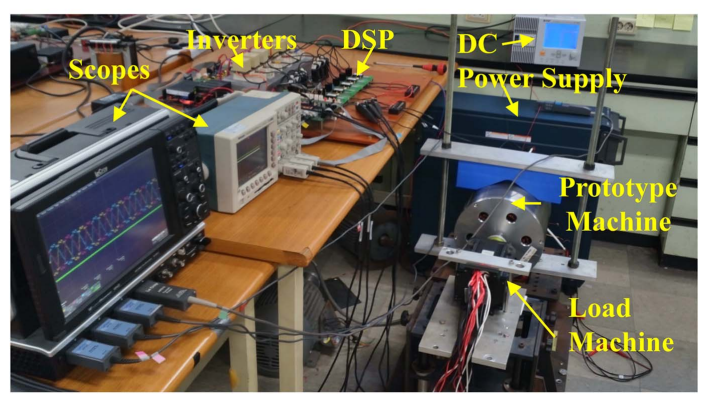

(d)

Fig. 7. (Color online) Experimental machine prototype components: (a) stator, (b) rotor laminations and (c) rotors. (d) Experimental setup.

to the load machine, DSP board, inverters, oscilloscopes and DC power supply.

Initially, no load test was carried out for the hybrid BLWRSM. The rotor was rotated at a base speed of $900 \mathrm{rpm}$ using a separate motor, and the back EMF of the machine was measured. The no-load test was used to validate the simulation results, because there is no way to measure the 


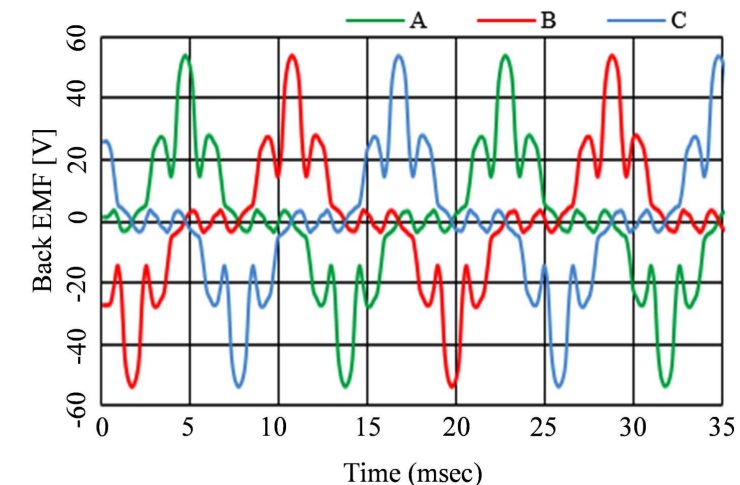

(a)

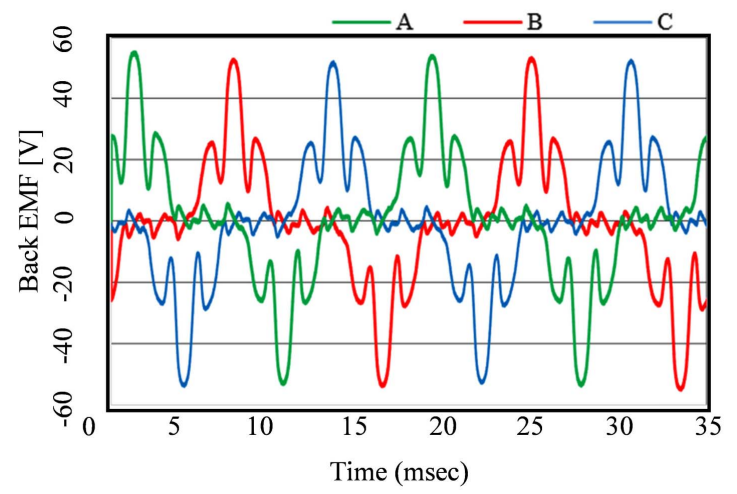

(b)

Fig. 8. (Color online) Back EMF profile of the machine: (a) simulated and (b) experimental.

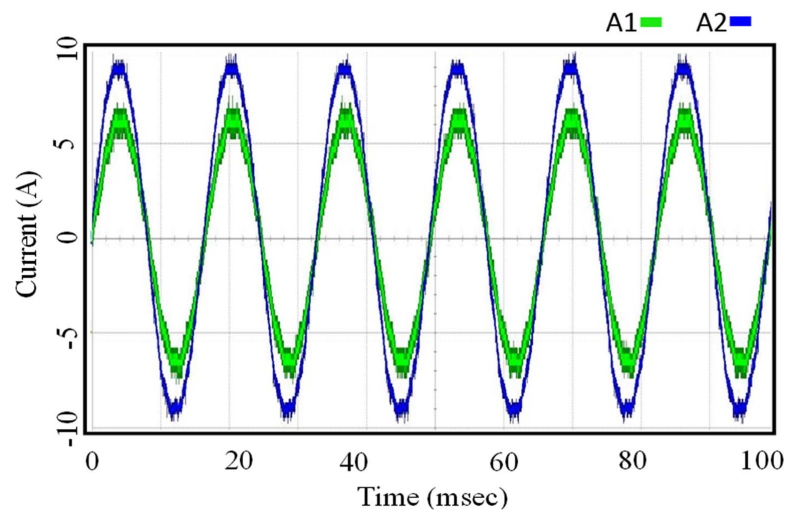

Fig. 9. (Color online) Input currents for the prototype machine.

rotor excitation current under load conditions in the experimental machine. As shown in Fig. 8(a), the simulated back EMF of the machine is 21.39 Vrms. Figure $8(\mathrm{~b})$ shows that the experimentally measured back EMF of the machine was $20.48 \mathrm{Vrms}$. These results showed a difference of $4.25 \%$ between the simulated and experimental back EMF of the machine.

After performing the no-load test of the machine, full-

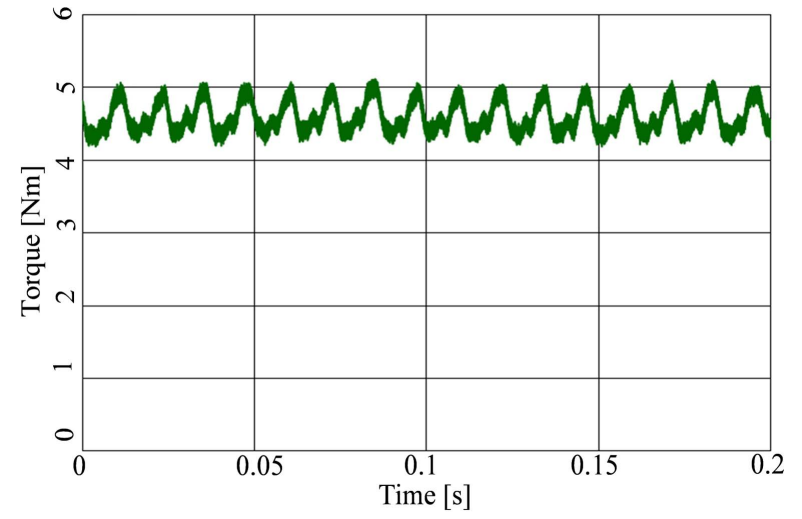

(a)

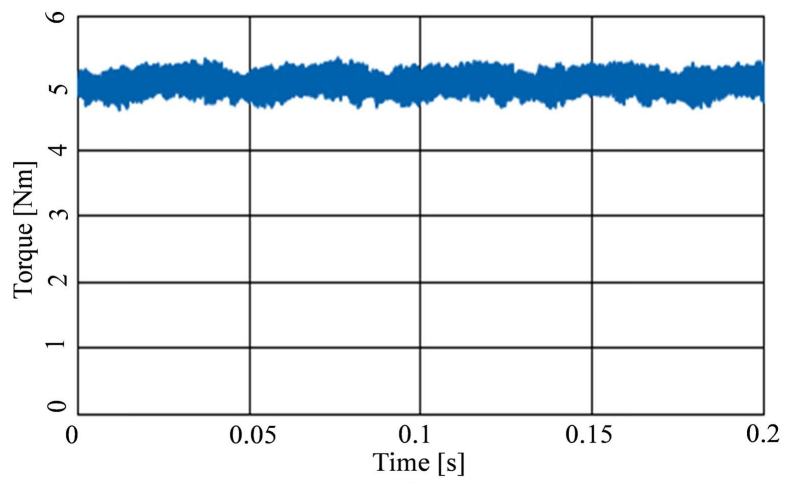

(b)

Fig. 10. (Color online) Electromagnetic torque of the prototype machines: (a) BL-WRSM and (b) hybrid BL-WRSM.

load tests were carried out for both the BL-WRSM and hybrid BL-WRSM. The stator currents were controlled at a proportion of 1:1.45 for the two stator windings of the machine i.e., $m=1.45$. Two inverters were used to feed the currents into the two windings of the machine. Machine currents under load conditions for phase-A with both portions are shown in Fig. 9. As shown, currents for the winding portion $\mathrm{A} 2$ were 1.45 times the magnitude of the currents of winding portion A1. The two currents have the same frequency and are in phase with each other.

The measured torque for the experimental machine with a PM assisted rotor is shown in Fig. 10. In the experimental analysis, torque ripples of about $18.5 \%$ were observed in the hybrid BL-WRSM compared to $19.2 \%$ in the BL-WRSM.

Table 2 compares the performances of the BL-WRSM and the hybrid BL-WRSM. The efficiency of the experimental hybrid BL-WRSM was measured to be $75.32 \%$. The efficiency and the torque ripple for the hybrid BLWRSM were slightly improved as compared to the BLWRSM. The reason behind this improvement is that the machine was less dependent upon the sub-harmonic excitation for the hybrid BL-WRSM due to the addition of 
Table 2. Comparison of Results.

\begin{tabular}{lccccc}
\hline \hline \multirow{2}{*}{ Parameter } & \multicolumn{2}{c}{ BL-WRSM } & & \multicolumn{2}{c}{ Hybrid BL-WRSM } \\
\cline { 2 - 3 } \cline { 5 - 6 } & Simulated & Experimental & & Simulated & Experimental \\
\hline Torque [Nm] & 4.74 & 4.60 & & 5.01 & 1.95 \\
Torque Ripple [\%] & 16.29 & 19.2 & - & 16.03 & - \\
Field current [A] & 4.9 & 73.23 & & 7.9 & 75.32 \\
Efficiency [\%] & 74.82 & & & 76.12 & - \\
\hline
\end{tabular}

PMs on the rotor, which directly interact with the fundamental components of the stator MMF.

\section{Conclusion}

In this paper, a PM-assisted hybrid BL-WRSM has been introduced that has hybrid excited rotors with windings and PMs. This topology has beneficial characteristics in terms of improved machine startup and torque density. Additionally, the proposed machine topology can be used for a wide range of machine speeds by employing the proposed flux weakening operation. A 2D-FEM analysis and experimental analysis were carried out to verify the performance of the proposed topology. The results demonstrate the effectiveness of the proposed machine.

\section{Acknowledgment}

This work was supported in part by the BK21PLUS Program through the National Research Foundation of Korea within the Ministry of Education, and in part by the Human Resources Program in Energy Technology through the Korea Institute of Energy Technology Evaluation and Planning within the Ministry of Trade, Industry and Energy, Korea, under Grant 20154030200730.

\section{References}

[1] Q. Ali, T. A. Lipo, and B. I. Kwon, IEEE Trans. Magn. 51, 11 (2015).

[2] I. Boldea, L. N. Tutelea, L. Parsa, and D. Dorrell, IEEE Trans. Ind. Electron. 61, 10 (2014).

[3] Y. Amara, L. Vido, M. Gabsi, E. Hoang, A. H. Ben Ahmed, and M. Lecrivain, IEEE Trans. Vehicular Technology 58, 5 (2009).

[4] D. Fodorean, A. Djerdir, I. A. Viorel, and A. Miraoui, IEEE Trans. Energy Convers. 22, 3 (2007).

[5] D. G. Dorrell, IEEE IECON (2012).

[6] G. H. Lee, H. H. Lee, and Q. Wang, J. Magn. 18, 4 (2013).

[7] C. S. Lee, J. H. Kim, and J. P. Hong, J. Magn. 20, 2 (2015).

[8] F. Zhang, Y. Zhao, G. Jia, and M. Lin, ICEMS (2014).

[9] G. Dajaku and D. Gerling, EDPC (2013).

[10] L. Sun, X. Gao, F. Yao, Q. An, and T. A. Lipo, ECCE (2014).

[11] G. Jawad, Q. Ali, T. A. Lipo, and B. I. Kwon, IEEE Trans. Magn. 52, 8106104 (2016). 